\title{
Tailoring long-range superlattice chirality in the molecular self- assemblies via weak fluorine-mediated interactions
}

Mykola Telychko ${ }^{1, \dagger}$, Lulu Wang ${ }^{1, \dagger}$, Chia-Hsiu Hsu ${ }^{2,3 \dagger}$, Guangwu Li ${ }^{1, \dagger}$, Xinnan Peng ${ }^{1}$, Shaotang Song ${ }^{1}$, Jie Su${ }^{1}$, Feng-Chuan Chuang ${ }^{2,3^{*}}$, Jishan $\mathrm{Wu}^{1, *}$, Ming Wah Wong ${ }^{1, *}$, Jiong $\mathrm{Lu}^{1,4^{*}}$

${ }^{1}$ Department of Chemistry, National University of Singapore, 3 Science Drive 3, Singapore 117543, Singapore

${ }^{2}$ Department of Physics, National Sun Yat-Sen University, Kaohsiung 80424, Taiwan.

${ }^{3}$ Physics Division, National Center for Theoretical Sciences, Taipei, 10617 Taiwan

${ }^{4}$ Centre for Advanced 2D Materials (CA2DM), National University of Singapore, 6 Science Drive 2, Singapore 117546, Singapore

$\dagger$ These authors contributed equally to this work (M. Telychko ${ }^{1 \dagger}$, L. Wang ${ }^{1 \dagger}$, C.-H. Hsu $\left.{ }^{2 \dagger}, \mathrm{G}^{-\mathrm{Li}^{1 \dagger}}{ }^{1 \dagger}\right)$ "Corresponding authors. Email: chmluj@nus.edu.sg (J. Lu); chmwmw@nus.edu.sg (M. W. Wong); chmwuj@nus.edu.sg (J. Wu); fchuang@mail.nsysu.edu.tw (F.C. Chuang);

\begin{abstract}
Controllable fabrication of the enantiospecific molecular superlattices is a matter of imminent scientific and technological interest. Herein, we demonstrate that long-range superlattice chirality in molecular self-assemblies can be tailored by tuning the interplay of weak intermolecular noncovalent interactions. Different chiral recognition patterns are achieved in the two molecular selfassemblies comprised by two molecular enantiomers with identical steric conformations, derived from the hexaphenylbenzene - the smallest star-shaped polyphenylene. By means of highresolution scanning tunneling microscopy measurements, we demonstrate that functionalization of
\end{abstract}


star-shaped polyphenylene with fluorine (F) atoms leads to the formation of molecular selfassemblies with the distinct long-range chiral recognition patterns. We employed the density functional theory calculations to quantify F-mediated lone pair F $\cdots \pi, \mathrm{C}-\mathrm{H} \cdots \mathrm{F}, \mathrm{F} \cdots \mathrm{F}$ interactions attributed to the tunable enantiospecific molecular self-organizations. Our findings underpin a viable route to tailor long-range chiral recognition patterns in supramolecular assemblies by engineering the weak non-covalent intermolecular interactions.

\section{Introduction}

Molecular chirality plays a detrimental role in a number of fundamental biochemical processes, including drug synthesis and in vivo stereochemical reactions. ${ }^{[1-2] . ~ T e c h n o l o g i c a l ~}$ importance of the chiral molecular nanostructures is exemplified by a wide range of their applications in non-linear chiroptics, enantioselective catalysis and enantiospecific sensing. ${ }^{[3-7]}$ Despite of increased scientific interest and research effort, the ability to controllably fabricate and precisely tailor the long-range chirality in the artificial functional nanostructures remains elusive.

Common strategy towards the fabrication of the enantiospecific chiral nanostructures relies on the self-organization of the molecular building blocks into extended two-dimensional (2D) assemblies with chiral recognition motifs, ${ }^{[8-12]}$ that can be tailored via tuning the interplay of various intermolecular interactions. It has been demonstrated that molecular self-organization and its associated enantiospecific architectures can be steered by manipulating a wealth of weak noncovalent interactions, including steric repulsion, ${ }^{[13-16]}$ hydrogen bonding, ${ }^{[17-19]}$ halogen bonding ${ }^{[20,21]}$ or dipolar ${ }^{[22]}$ interactions. In addition, metal-center mediated covalent bonding ${ }^{[23-25]}$ and molecule-substrate interactions have also been recently utilized to create a variety of the enantiospecific nanostructures. ${ }^{[26-30]}$

The three-dimensional (3D) character of non-planar molecules with the presence of heteroatoms (e.g. oxygen, nitrogen or fluorine) in the molecular backbone offers multiple weak intermolecular interactions that can be exploited to fabricate plethora of feasible self-organization motifs with the possibility to engineer the advanced chiral supramolecular architectures. Future progress in the fabrication of functional chiral supramolecular nanostructures hinges on fundamental understanding of the correlation between weak intermolecular non-covalent interactions and the assembled chiral motifs. 
Fluorine-mediated (F-mediated) weak interactions in organofluorine compounds and their role in steering molecular assemblies have remained most elusive among various non-covalent interactions reported to date. ${ }^{[31,32]}$ On one hand, the absence of a pronounced sigma-hole, a fingerprint of a heavier halogen atom (i.e. bromine, chlorine and iodine), precludes the $\mathrm{F}$ from participating in the halogen bonding guided molecular self-assemblies. On the other hand, $\mathrm{F}$ atom also acts as a relatively weak hydrogen-bond acceptor and thus exhibits a significantly lower trend towards the participation in hydrogen bonding interactions compared to that of nitrogen or oxygen atoms. ${ }^{[33,34]}$ It has been recently demonstrated that weak attractive F-based interaction becomes one of dominant forces between planar fluorinated aromatic molecules, particularly in the absence of other types of competing intermolecular interactions. ${ }^{[35,36]}$ The presence of electron-deficient aromatic rings in non-planar organofluorine compounds may also lead to an emergence of $F \cdots \pi$ stabilizing interactions. ${ }^{[37,38]}$ The role of F-mediated intermolecular interactions in organization of organofluorine enantiomers into two-dimensional enantiospecific assemblies remains largely unexplored.

Microscopic knowledge of the long-range chiral recognition in the molecular selfassemblies driven by multiple types of weak intermolecular interactions, particularly F-mediated interactions, is still not well understood. Herein, we demonstrate a tunable long-range chiral recognition of the supramolecular assemblies via utilization of the weak F-mediated interactions. Specifically, we studied two distinct self-assemblies formed by two precursor molecules, derived from the hexaphenylbenzene. Both precursor molecules possess identical centrosymmetric $\mathrm{D}_{6}$ symmetry and prochiral character due to the sterically-driven rotation of the six dimethyl-phenyl rings, but differ by fluorine (F) or hydrogen $(\mathrm{H})$ termination of a dimethyl-phenyl rings (Fig 1a, b). We have determined the chirality of individual molecular building blocks in the self-assembled molecular superlattices using high-resolution scanning tunneling microscopy (STM). It is found that the presence weak F-mediated $\mathrm{C}-\mathrm{H} \cdots \mathrm{F}$ and lone pair $\mathrm{F} \cdots \pi$ intermolecular interactions leads to the dramatically different chiral recognition motifs of the studied supramolecular selfassemblies. 


\section{Results \& Discussion}

HPB and F-HPB enantiomers. Two molecular precursors were chosen as building blocks to explore their supramolecular self-assemblies on $\operatorname{Ag}(111)$ surface. The first molecular precursor, namely 3',4',5',6'-tetrakis(3,5-dimethylphenyl)-3,3',5,5'"-tetramethyl-1,1':2',1'-terphenyl, $\mathrm{C}_{54} \mathrm{H}_{44}$ (entitled as HPB) exhibits a $\mathrm{D}_{6}$ symmetry with six dimethylphenyl rings attached to the core benzene ring via sigma bonds. Each dimethyl-phenyl ring contains one phenyl rings and two methyl groups attached at 3,5 positions (Fig. 1a). The second molecular precursor, namely 4,4"difluoro-3',4',5',6'-tetrakis(4-fluoro-3,5-dimethylphenyl)-3,3",5,5"-tetramethyl-1,1':2', 1"-

terphenys, $\mathrm{C}_{54} \mathrm{H}_{48} \mathrm{~F}_{6}$ (entitled as F-HPB) (Fig. 1b), is derived from HPB via the substitution of the $\mathrm{H}$ atoms at apices of the dimethyl-phenyl rings with $\mathrm{F}$ atoms. The synthetic routes of the HPB and F-HPB compounds has been discussed in detail in our previous reports. ${ }^{[39,40]}$

HPB and F-HPB molecules possess several structural motifs and associated intramolecular electrostatic potential profiles, which are expected to harbor multiple weak intermolecular interactions guiding molecular self-assembling process. Firstly, an intrinsic steric hindrance within HPB and F-HPB molecules manifested by the rotation of the dimethyl-phenyl rings around their sigma chemical bonds renders their highly non-planar conformations. ${ }^{[47,48]}$ Upon their adsorption on the $\operatorname{Ag}(111)$ substrate, the clockwise or counterclockwise orientations of the phenyl rings is expected to yield two mirror-symmetric left-handed (L) and right-handed (R)- enantiomeric configurations of both HPB and F-HPB molecules. It has been demonstrated that the steric repulsion and aromatic interactions between non-planar molecules can govern the formation of the enantiopure supramolecular monolayers. ${ }^{[41,13,15]}$ Secondly, the substitution of H (in HPB) by electronegative F (in F-HPB), leads to a notable variation in the intramolecular charge redistribution. The electrostatic potential (ESP) map of F-HPB (Fig. 1d) reveals an electron-rich belt region in vicinity of the $\mathrm{F}$, whilst dimethyl-phenyl ring hosts a positive charge. Interactions between these electron-deficient aromatic rings and electronegative $\mathrm{F}$ atoms may lead to an emergence of attractive $\mathrm{F} \cdots \pi$ electrostatic interactions. ${ }^{\left[{ }^{37,38]}\right.}$ Last but not least, their intrinsic $\mathrm{D}_{6}$ symmetry of both HPB and F-HPB molecules eliminates the presence of a global dipole moment of individual molecules, which rules out the possible participation of long-range dipolar interactions in the self-assembling process. ${ }^{[22]}$ Therefore, on-surface self-organization of the HPB and F-HPB molecules is expected to be steered by the interplay of multiple intermolecular weak 
interactions including repulsive steric interactions and weak intermolecular interactions such as $\mathrm{H}$ bonding and $\pi-\pi$ (in both HPB and F-HPB assembly). The presence of lone pair F $\cdots \pi, \mathrm{C}-\mathrm{H} \cdots \mathrm{F}$ and $\mathrm{F} \cdots \mathrm{F}$ interactions in F-HPB may result in the formation of new self-assembled superstructures different from HPB. In addition, a rational design of HPB and F-HPB enantiomers with identical steric conformations allows to quantify the impact of a weak F-mediated interactions in the assembly of the long-range chiral recognition motifs.

STM imaging of HPB and F-HPB self-assemblies. Our STM imaging captures HPB and F-HPB molecular self-assemblies on $\mathrm{Ag}(111)$ with remarkably different periodicities, as revealed in the representative large-scale STM images (Fig. 2a,b) and the corresponding Fourier-transformed STM images (insets in Fig. 2a,b). The HPB-based self-assembly exhibits a highly periodic and defect-free pattern, characterized by a $\sqrt{3} \times \sqrt{3}$ elementary unit cell with a lattice constant of $a=$ $b=23.19 \AA$ (Fig. 2a). In contrast, the F-HPB self-assembly is presented by a $2 \times 1$ supercell lattice, with a unit cell constant of $a=27.30 \AA, b=14.02 \AA$ (Fig. 2b).

Fig. 3c,d shows the representative high-resolution STM images of the HPB and F-HPB selfassemblies, respectively. A detailed image analysis reveals that each molecule exhibits a six-fold symmetry, as it is comprised by six protrusions arranged in a hexagonal-like manner (Fig. 3 a,b). These bright spots are attributed to protruding methyl $\left(-\mathrm{CH}_{3}\right)$ groups attached to dimethyl-phenyl rings. Furthermore, it is noted that individual HPB and F-HPB molecules exhibit an overall clockwise or counterclockwise appearance due to their expected L- or R- handed enantiomeric configurations. Such a characteristic STM appearance allows for the determination of the chirality of each molecule in HPB and F-HPB self-assemblies. L- and R-handed chiral enantiomers are overlaid by grey- and pink-colored molecules, respectively (Fig. 3 e,f). It is evident that HPBbased self-assembly adopts a $\sqrt{3} \times \sqrt{3}$ unit cell, which is comprised by four L-handed enantiomers and two R-handed enantiomers (Fig. 3e). The presence of the dominant R-handed enantiomers ( $\mathrm{L}: \mathrm{R}$ ratio is determined to be 1:2) in these HPB domains endows a net R-handed domain chirality. Other domains with dominant L-handed enantiomers revealing a net L-handed chirality is shown in Supplementary Fig. S1. In contrast to HPB, the F-HPB superstructure with a $2 \times 1$ periodicity is presented by alternating homochiral rows comprised solely by R- or L-handed F-HPB enantiomers as shown in Fig. 3 b (also Supplementary Figs. S2 and S3). 
Intermolecular interactions in HPB and F-HPB self-assemblies. Based on these observations, we performed DFT SLAB calculations using $\sqrt{3} \times \sqrt{3}$ and $2 \times 1$ supercell structures of both HPB and F-HPB assemblies including the clockwise and counterclockwise rotations of the dimethylphenyl rings supported by four-layered slab of $\mathrm{Ag}(111)$. In line with experimental observations, we found that $\sqrt{3} \times \sqrt{3}$ HPB $[(2 \times 1)$ F-HPB] structures are energetically more favorable than $2 \times 1$ HPB $[(\sqrt{3} \times \sqrt{3})$ F-HPB] (see energies in Supplementary Tables S1 and S2). The relaxed $\mathrm{Ag}(111)$-supported elementary unit cells of $\sqrt{3} \times \sqrt{3}$ HPB and $(2 \times 1)$ F-HPB obtained using DFT GGA-PBE functional are shown in Fig. 4a and Fig. 4b, respectively. The unit cell lattice constants of $\sqrt{3} \times \sqrt{3} \mathrm{HPB}$ and $2 \times 1$ of F-HPB are determined to be $a=b=23.35 \AA$ and $a=26.52 \AA$, $b=14.80 \AA$ respectively, in good agreement with the experimentally determined values, which further validates our DFT-relaxed models.

The landscape of charge density difference (CDD) (in Fig. S4) of both HPB and F-HPB assemblies exhibits strong (weak) intensity in between heterochiral (homochiral) enantiomers, suggesting that intermolecular organization is steered presumably by steric-repulsive and attractive interactions between heterochiral enantiomers. Furthermore, the calculated values of the cohesive energies of the heterochiral enantiomers are significantly lower compared to that of homochiral enantiomers in both HPB and F-HPB structures (see Supplementary Table S2). To get further insight into the intermolecular interactions in HPB and F-HPB and energetic gain of the heterochiral packing, topological analysis of electron density based on Bader's quantum theory of atoms in molecules $(\text { QTAIM })^{[42]}$ was performed. The nature and strength of non-covalent interaction are characterized by the electron density $(\rho(r))$ and its Laplacian $(\nabla 2 \rho(r))$ at the bond critical point (BCP).

Our AIM analysis of the HPB $\sqrt{3} \times \sqrt{3}$ structure reveals the presence of multiple attractive C$\mathrm{H} \cdots \pi^{[43-46]}$ stabilizing interactions (denoted as $a_{1}-a_{5}$ in Fig. 4b), involving $\mathrm{H}$ atoms at apices of the dimethyl-phenyl ring ( $a_{2}$ and $\left.a_{5}\right)$ or methyl C-H $\left(a_{1}, a_{3}\right.$ and $\left.a_{4}\right)$ and $\pi$-electrons of dimethylphenyl rings. For the aromatic C-H's (i.e. $a_{2}$ and $a_{5}$ ), the $\mathrm{C}-\mathrm{H} \cdots \pi$ interactions are also called edgeto-face aromatic-aromatic interactions. ${ }^{[47]}$ The $\pi \cdots \pi$ stacking interaction ${ }^{[47]}$ between two units of dimethyl-phenyl ring $\left(b_{1}\right)$ is also observed (Fig. 4b). Although a quantitative comparison of the strength of these interactions is not feasible, we note that the heterochiral packing (red panel in Fig. 4b) contains more attractive $\mathrm{C}-\mathrm{H} \cdots \pi$ interactions $\left(a_{1}-a_{5}\right)$ than homochiral packing, which 
likely renders heterochiral HPB packing energetically more favorable (i.e. a lower cohesive energy). Therefore, HPB enantiomers tend to maximize the number of the heterochiral packing motifs during the self-assembling process, giving rise to the observed $\sqrt{3} \times \sqrt{3}$ reconstruction, wherein one L- (or R-) handed enantiomer is surrounded by six (i.e. maximal number) R- (or L-) handed enantiomers.

Analogously, the heterochiral F-HPB packing (red panel in Fig. 4d) also features attractive C$\mathrm{H}^{\cdots} \pi$ interactions (denoted as $a_{1}$ and $a_{2}$ in Fig. 4d), involving methyl C-H and $\pi$ system of the dimethyl-phenyl rings. Besides that, both homochiral and heterochiral F-HPB packings reveal abundant weak $\mathrm{C}-\mathrm{H} \cdots \mathrm{F}$ hydrogen bonds $\left(c_{1}-c_{4}\right)$, involving methyl $\mathrm{C}-\mathrm{H}$ and $\mathrm{F}$ atoms. Interestingly, we also identified the presence of lone pair F $\cdots \pi$ ( $d_{1}$ and $d_{2}$ in red panel in Fig. $\left.4 \mathrm{~d}\right)$ interactions between heterochiral F-HPB enantiomers. These interactions were reported to take place between negatively charged $\mathrm{F}$ atoms and positively charged aromatic rings, ${ }^{[37,48-50]}$ although existence of these interactions in 2D supramolecular assemblies has not been experimentally observed to date. The formation of F-HPB self-assemblies is likely steered by cooperative attractive lone pair $\mathrm{F} \cdots \pi$, $\mathrm{C}-\mathrm{H} \cdots \mathrm{F}$ and $\mathrm{C}-\mathrm{H} \cdots \pi$ interactions between heterochiral enantiomers, whilst the presence of the notable attractive $\mathrm{C}-\mathrm{H} \cdots \mathrm{F}$ interactions between homochiral F-HPB enantiomers facilitates the formation of enantiopure chains comprising the $2 \times 1$ superstructure. The existence of various weak non-covalent interactions is further supported by non-covalent interactions (NCI) index plots $^{[51]}$ (see Supplementary Figs. S5 and S6). The result of NCI analysis transforms NCI from the reduced density gradient into the surface, in which color represents the nature and strength of the non-covalent interaction.

In summary, we have demonstrated that the long-range chiral recognition patterns of the two distinct supramolecular self-assemblies can be steered by tuning the interplay of multiple types of weak non-covalent interactions. The presence of weak F-mediated interactions in F-HPB selfassemblies renders a distinct long-range chiral recognition motif with a $2 \times 1$ superlattice, in stark contrast to HPB enantiomers that adopt a $\sqrt{3} \times \sqrt{3}$ chiral superstructure. Our findings underpin a viable route towards a rational design and fabrication of the chiral recognition motifs in the functional supramolecular nanostructures via tuning the interplay of weak non-covalent interactions. 
Acknowledgements: J. Lu acknowledges the support from MOE grants (MOE2019-T2-2-044 and R-143-000-B58-114). M. Telychko acknowledges support from A*STAR AME YIRG grant (Project No. A20E6c0098, R-143-000-B71-305). F.C.C. acknowledges support from the National Center for Theoretical Sciences and the Ministry of Science and Technology of Taiwan under grant nos. MOST-107-2628-M-110-001-MY3. He is also grateful to the National Center for Highperformance Computing for computer time and facilities. L. Wang thanks Applied Materials for the Ph.D. scholarship.

Author contributions: M.T and J.L. conceived and designed the experiments. M.T. performed all the experiment related to the fabrication of HPB/F-HPB self-assemblies, their STM imaging and data analysis. L. W. performed DFT, AIM and NCI calculations under supervision of M. W. W. C.-H. Hsu performed DFT calculations under supervision of F. C. Chuang. G. L. performed synthesis of HPB and F-HPB compounds under supervision of J. W. X.P, J.S and S.S. participated in a scientific discussion. The manuscript was written by M.T. and L.J. with contributions of all co-authors.

Competing financial interests: The authors declare that they have no competing interests. 

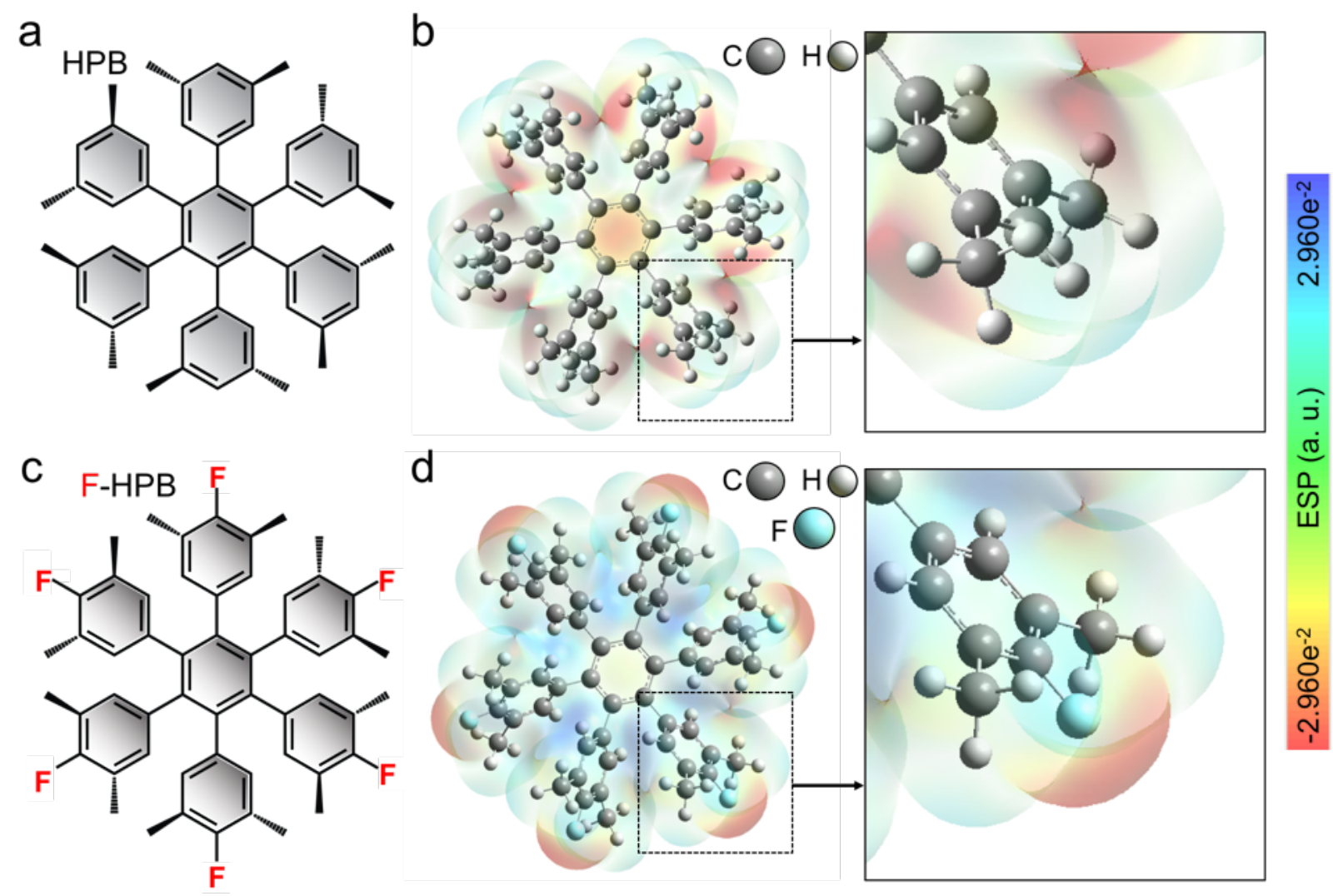

Fig. 1: Chemical Structure and electrostatic potential (ESP) maps of HPB and F-HPB precursor molecules. (a, b) chemical structure (a, c) and ball-and-stick model $(\mathbf{b}, \mathbf{d})$ overlaid with map of the electrostatic potential of HPB and F-HPB molecule, respectively. Note: ESP is plotted at isovalue of 0.001 a.u., with red and blue color represent negative and positive ESP value, respectively. ESP reveals the electron rich area in the vicinity of $\mathrm{F}$ atom within an individual FHPB molecule. 

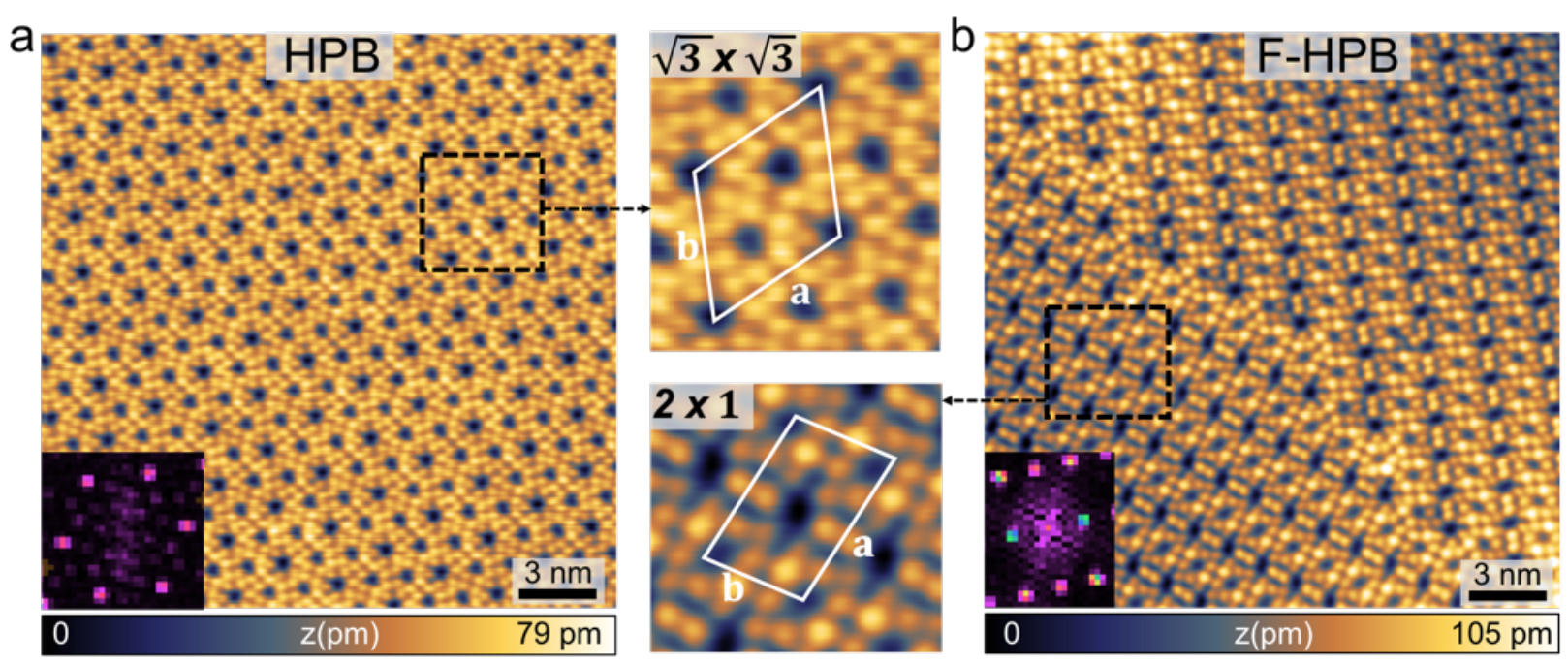

Fig. 2: Supramolecular self-assemblies of HPB and F-HPB precursors on $\operatorname{Ag}(111)$ substrate. (a, b) Large-scale STM images of (panel a) the HPB and (panel b) F-HPB self-assemblies on Ag(111) substrate. Insets in panels $\mathbf{a}$ and $\mathbf{b}$ are the corresponding Fourier transformed images, which reveal the characteristic spots associated with the $\sqrt{3} \times \sqrt{3}$ and $2 \times 1$ elementary unit cells of HPB and F-HPB self-assemblies, respectively. 

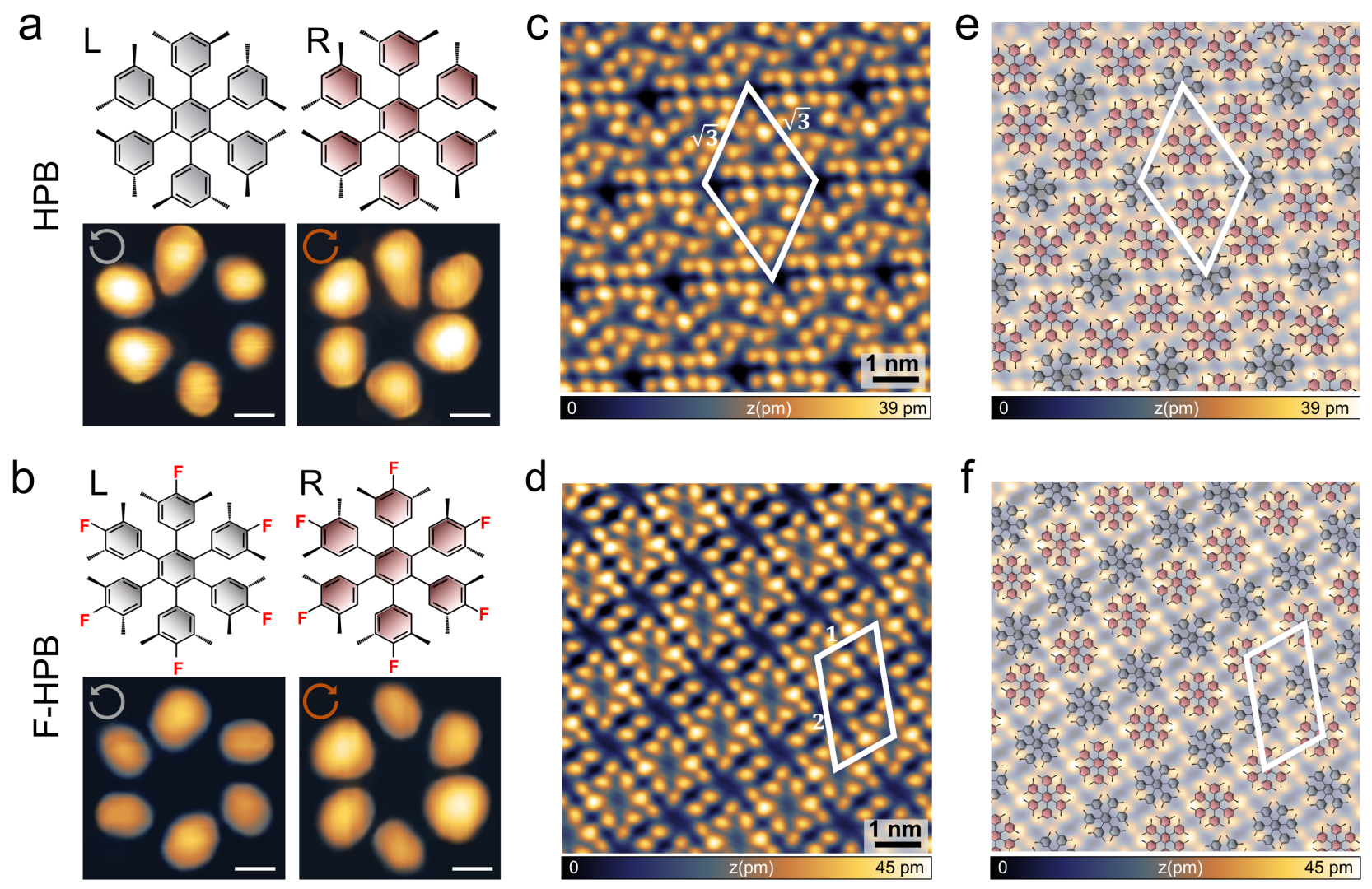

Fig. 3: (a, b) STM images of the two L- and R-handed HPB (a) and F-HPB (b) enantiomers. The grey and pink molecular chemical structures represent the enantiomers with L- and R chirality, respectively. (c,d) representative STM images of HPB and F-HPB self-assemblies (e,f) STM images in (c,d) overlaid the L- and R-handed molecular structures to illustrate the long-range chiral motifs of HPB and F-HPB self-assemblies. 

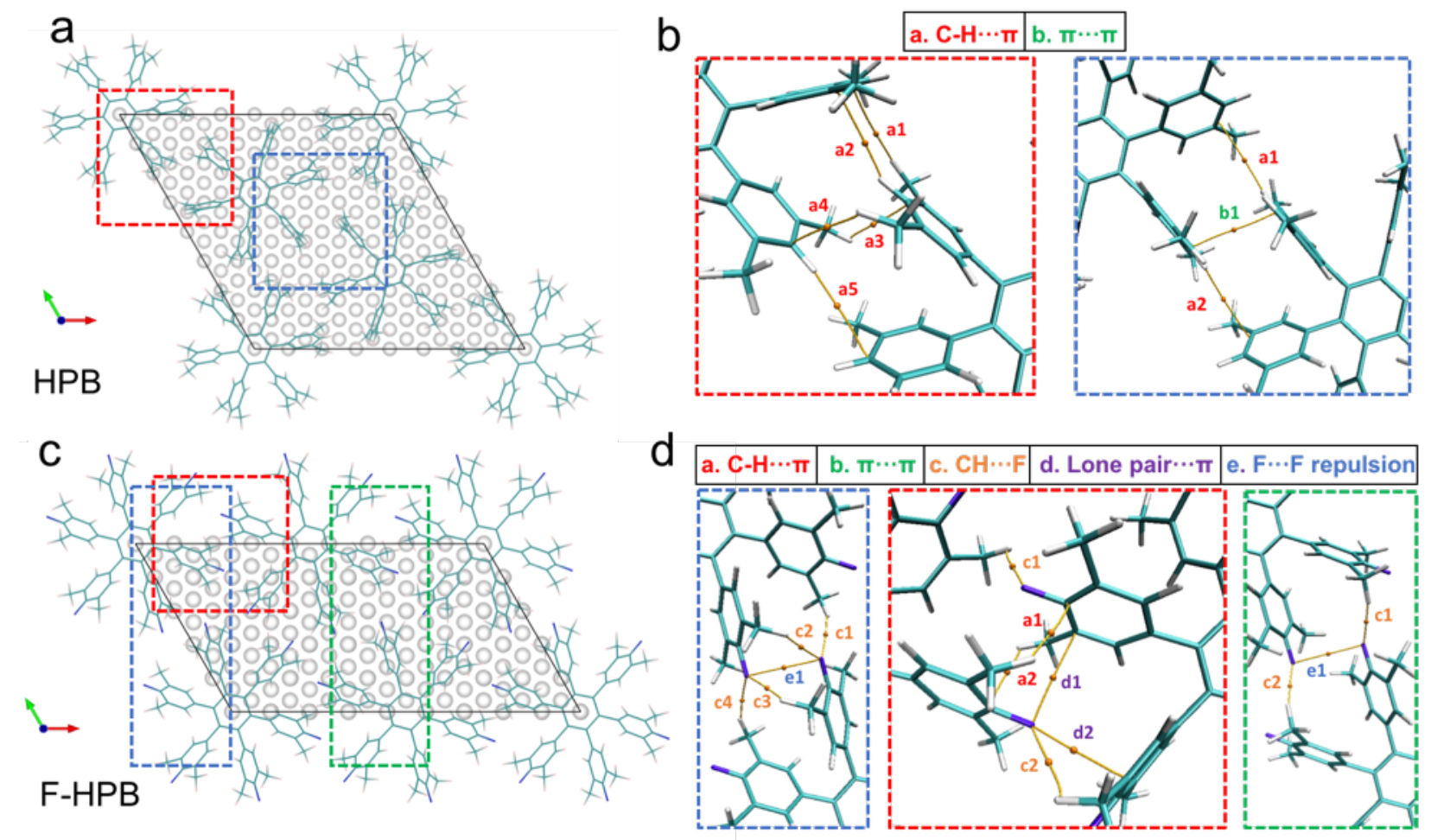

Fig. 4. Analyzing weak non-covalent interactions in the HPB and F-HPB assemblies based on AIM analysis. (a) DFT-relaxed $\sqrt{3} \times \sqrt{3}$ unit cell of the HPB self-assembly. (b) Magnified view of the area marked by red (heterochiral enantiomers) and blue (homochiral enantiomers) rectangle in panel a reveals multiple weak C-H $\cdots \pi\left(a_{1}-a_{5}\right)$ and $\pi \cdots \pi\left(b_{1}\right)$ interactions between heterochiral HPB enantiomers. (c) DFT-relaxed $2 \times 1$ unit cell of the F-HPB self-assembly. (d) Magnified view of the area marked by blue (homochiral enantiomers) and red (heterochiral enantiomers) in panel b reveals a set of weak non-covalent interactions including $\mathrm{C}-\mathrm{H} \cdots \pi\left(\mathrm{a}_{1}\right.$ and $\left.\mathrm{a}_{2}\right), \mathrm{C}-\mathrm{H} \cdots \mathrm{F}$ $\left(\mathrm{c}_{1}-\mathrm{c}_{4}\right), \mathrm{F}$ lone pair $\cdots \pi\left(\mathrm{d}_{1}\right.$ and $\left.\mathrm{d}_{2}\right)$ and $\mathrm{F} \cdots \mathrm{F}$ repulsion $\left(\mathrm{e}_{1}\right)$. 


\section{References}

[1] W. H. Brooks, W. C. Guida, K. G. Daniel, Curr Top Med Chem. 2011, 11, 760-770

[2] J. Cohen, Science 1995, 267, 1265.

[3] Z. Chen, X. Lu, Nano Ex. 2020, 1, 032002.

[4] K. Soai, T. Shibata, H. Morioka, K. Choji, Nature 1987, 329, 712.

[5] M. Heitbaum, F. Glorius, I. Escher, Angew. Chem. Int. Ed. 2006, 45, 4732-4762.

[6] C. Baleizão, H. Garcia, Chem. Rev. 2006, 106, 3987-4043.

[7] J. A. Switzer, H. M. Kothari, P. Poizot, S. Nakanishi, E. W. Bohannan, Nature 2003, 425, 490-493.

[8] R. Raval, Chem. Soc. Rev. 2009, 38, 707-721.

[9] K.-H. Ernst, Phys. Status Solidi B 2012, 249, 2057-2088.

[10] T. Chen, D. Wang, L.-J. Wan, Natl Sci. Rev. 2015, 2, 205-216.

[11] Y. Fang, E. Ghijsens, O. Ivasenko, H. Cao, A. Noguchi, K. S. Mali, K. Tahara, Y. Tobe, S. De Feyter, Nat. Chem. 2016, 8, $711-717$ (2016).

[12] K. Tahara, H. Yamaga, E. Ghijsens, K. Inukai, J. Adisoejoso, M. O. Blunt, S. De Feyter, Y. Tobe, Nat. Chem. 2011, 3, 714-719.

[13] W. Xiao, X. Feng, P. Ruffieux, O. Gröning, K. Müllen, R. Fasel, J. Am. Chem. Soc. 2008, 130, 8910-8912.

[14]A. Mairena, J. I. Mendieta, O. Stetsovych, A. Terfortc, I. G. Starád, I. Starý, P. Jelínek, K.-H. Ernst, Chem Comm. 2019, 55, 10595-10598.

[15] R. Fasel, M. Parschau, K.-H. Ernst, Nature 2006, 439, 449-452.

[16] M.-C. Blüm, E. Ćavar, M. Pieta, F. Pat they, W. D. Schneider, Angew. Chem. Int. Ed. 2005, 44, 5334-5337.

[17]M. Stöhr, S. Boz, M. Schär, M.-T. Nguyen, C. A. Pignedoli, D. Passerone, W. Bernd Schweizer, C. Thilgen, T. A. Jung, F. Diederich, Angew. Chem. Int. Ed. 2011, 50, 9982-9986.

[18] K. Miao, Y. Hu, L. Xu, M. Dong, J. Wu, X. Miao, W. Deng, Phys. Chem. Chem. Phys. 2018, 20, 11160-11173.

[19] H. Cao, S. De Feyter, Nat. Comm. 2018, 9, 3416.

[20] Gutzler, O. Ivasenko, C. Fu, J. L. Brusso, F. Rosei, D. F. Perepichka. Chem. Commun. 2011, 47, 9453-9455 (2011). 
[21] J. K. Yoon, W. Son, H. Kim, K.-H. Chung, S. Han, S.-J Kahng, Nanotechnology 2011, 22, 275705.

[22] H. Kong, Y. Qian, X. Liu, X. Wan, S. Amirjalayer, H. Fuchs, Angew. Chem. Int. Ed. 2020, $59,182-186$.

[23]Zhang, Z. Gong, K. Sun, R. Duan, P. Ji, L. Li, C. Li, K. Müllen, L Chi, J. Am. Chem. Soc. 2016, 138, 11743-11748.

[24] N. Merino-Díez, M. S. G. Mohammed, J. Castro-Esteban, L. Colazzo, A. Berdonces-Layunta, J. Lawrence, J. I. Pascual, D. G. de Oteyza, D. Peña Chem. Sci. 2020, 11, 5441-5446.

[25] L. L. Patera, Z. Zou, C. Dri, C. Africh, J. Repp, G. Comelli, Phys. Chem. Chem. Phys. 2017, $19,2460$.

[26] Wang, T., Lv, H., Huang, J. et al. Nat. Commun. 2019, 10, 4122.

[27] H. Chen, L. Tao, D. Wang, Z.-Y. Wu, J.-L. Zhang, S. Gao, W. Xiao, Shixuan Du, K.-H. Ernst, H.-J. Gao, Angew. Chem. Int. Ed. 2020, 59, 17413-17416 (2020).

[28] J. Park, J.-H. Kim, S. Bak, K. Tahara, J. Jung, M. Kawai, Y. Tobe, Y. Kim, Angew. Chem. Int. Ed. 2019, 58(28), 9611-9618.

[29] W. Xiao, H. K. Ernst, K. Palotas, K. et al, Nature Chem. 2016, 8, 326-330.

[30] S. Stolz, A. V. Yakutovich, J. Prinz, T. Dienel, C. A. Pignedoli, H. Brune, O. Gröning, R. Widmer, Angew. Chem. Int. Ed. 2020, 132, 18336-18340.

[31] R. Berger, G. Resnati, P. Metrangolo, E. Weber, J. Hulliger, Chem. Soc. Rev. 2011, 40, $3496-$ 3508.

[32] D. O'Hagan, Chem. Soc. Rev. 2008, 37, 308-319.

[33] P. Zhou, J. Zou, F. Tian, Z. Shang, J. Chem. Inf. Model. 2009 49, 2344-2355.

[34]H.-J. Schneider, Chem. Sci., 2012, 3, 1381.

[35] J. Niederhausen, Y. Zhang, F. Cheenicode Kabeer, Y. Garmshausen, B. M. Schmidt, Y. Li, K.-F. Braun, S. Hecht, A. Tkatchenko, N. Koch, S.-W. Hla, J. Phys. Chem. C 2018,122, 18902-18911.

[36] S.Kawai, A. Sadeghi, F. Xu, L. Peng, A. Orita, J. Otera, S. Goedecker, E. Meyer, ACS Nano, 2015, 9, 2574-2583.

[37]P. Li, J. M Maier, E. C Vik, C. J .Yehl, B. E. Dial, A. E. Rickher, M. D. Smith, P. J Pellechia, K. D. Shimizu, Angew. Chem. Int. Ed. 2017, 56, $7209-7212$ (2017). 
[38] M. G. Holl, M. D. Struble, P. Singal, M. A. Siegler, T. Lectka, Angew. Chem. Int. Ed. 2016, $55,8266-8269$.

[39] M. Telychko, G. Li, P. Mutombo, D. Soler-Polo, X. Peng, J. Su, S. Song, M. Joo Koh, M. Edmonds, P. Jelínek, J. Wu, J. Lu, Sci. Adv. 2021, 7, eabf0269.

[40] G. Li, Y. Han, Y. Zou, J. J. C. Lee, Y. Ni, J. Wu, Angew. Chem. Int. Ed. 2019, 58, 1431914326.

[41]M. Treier, P. Ruffieux, P. Gröning, S. Xiao, C. Nuckollsb, Roman Fasel Chem. Commun. 2008, 38, 4555-4557.

[42] E. R. Johnson, S. Keinan, P. Mori-Sánchez, J. Contreras-García, A. J. Cohen, W. Yang, J. Am. Chem. Soc. 2010, 132, 6498-6506.

[43] J. Ran, M. W. Wong, J. Phys. Chem. A 2006, 110, 9702-9709.

[44] M. Nishio, M. Hirota, Y. Umezawa, Wiley-VCH, New York 1998.

[45] P. Hobza, Z. Havlas, Chem. Rev. 2000, 100, 4253-4264.

[46] K. S. Kim, T. Parakeshwar, J. Y. Lee, Chem. Rev. 2000, 100, 4145-4186.

[47] E. C. Lee, D. Kim, P. Jurecka, P. Tarakeshwar, P. Hobza, K. S. Kim, J. Phys. Chem. A 2007, $111,3446-3457$.

[48] T. J. Mooibroek, P. Gamez, J. Reedijk, Cryst. Eng. Comm. 2008, 10, 1501-1515.

[49] J. Novotny, S. Bazzi, R. Marekac, J. Kozelka, Phys. Chem. Chem. Phys. 2016, 18, 19472 (2016).

[50] J. Ran, P. Hobza, J. Chem. Theory Comput. 2009, 5, 1180-1185 (2009).

[51] E. R. Johnson, S. Keinan, P. Mori-Sánchez, J. Contreras-García, A. J. Cohen, W. Yang, J. Am. Chem. Soc. 2010, 132, 6498-6506. 


\section{Supplementary information}

\section{Tailoring long-range superlattice chirality in the molecular self- assemblies via weak fluorine-mediated interactions}

Mykola Telychko ${ }^{1, \dagger}$, Lulu Wang ${ }^{1, \dagger}$, Chia-Hsiu Hsu ${ }^{2,3 \dagger}$, Guangwu Li ${ }^{1, \dagger}$, Xinnan Peng ${ }^{1}$, Shaotang Song $^{1}$, Jie Su${ }^{1}$, Feng-Chuan Chuang ${ }^{2,3 *}$, Jishan $\mathrm{Wu}^{1, *}$, Ming Wah Wong ${ }^{1, *}$, Jiong $\mathrm{Lu}^{1,4^{*}}$

${ }^{1}$ Department of Chemistry, National University of Singapore, 3 Science Drive 3, Singapore 117543, Singapore

${ }^{2}$ Department of Physics, National Sun Yat-Sen University, Kaohsiung 80424, Taiwan.

${ }^{3}$ Physics Division, National Center for Theoretical Sciences, Taipei, 10617 Taiwan

${ }^{4}$ Centre for Advanced 2D Materials (CA2DM), National University of Singapore, 6 Science Drive 2, Singapore 117546, Singapore

$\dagger$ These authors contributed equally to this work (M. Telychko ${ }^{1 \dagger}$, L. Wang ${ }^{1 \dagger}$, C.-H. Hsu ${ }^{2 \dagger}$, G. $^{2} \mathrm{Li}^{1 \dagger}$ ) *Corresponding authors. Email: chmluj@nus.edu.sg(J.Lu); chmwmw@nus.edu.sg (M. W.

Wong); chmwuj@nus.edu.sg (J. Wu); fchuang@mail.nsysu.edu.tw (F.C. Chuang); 


\section{Experimental Procedures}

Sample preparation. The $\mathrm{Ag}(111)$ single crystal (MaTeck $\mathrm{GmbH})$ was cleaned by multiple cycles of $\mathrm{Ar}^{+}$sputtering and annealing. A Knudsen cell (MBE-Komponenten $\mathrm{GmbH}$ ) was used to sublimate the HPB and F-HPB precursor molecules at $160^{\circ} \mathrm{C}$ and $180{ }^{\circ} \mathrm{C}$, respectively, which are deposited onto freshly prepared $\operatorname{Ag}(111)$ surface held at room temperature under ultrahigh vacuum conditions (base pressure $<1 \times 10^{-9} \mathrm{mbar}$ ). Subsequently, the sample was transferred into the STM head held at $4.5 \mathrm{~K}$ for STM imaging.

STM/nc-AFM measurements: The STM experiments were performed in ultra-high vacuum conditions (base pressure $<5 \times 10^{-11} \mathrm{mbar}$ ) at $4.4 \mathrm{~K}$ using a commercial Omicron low temperature STM system.

DFT method: All periodic slab calculations were performed using the DFT framework as implemented in the Vienna ab initio simulation package VASP using PBE functional ${ }^{[52]}$ and a projector augmented plane wave basis set $(\mathrm{PAW})^{[53]}$ with an energy cutoff of $400 \mathrm{eV}$. The van der Waals interactions were corrected using DFT-D3 scheme by Grimme ${ }^{[54]}$. Energies and geometry optimizations were converged to $10^{-5} \mathrm{eV}$. The slab model containing four atomic layers of $\mathrm{Ag}(111)$ was utilized and the bottom two layers were frozen. To create the molecular structures, a vacuum of $20.0 \AA$ was added above the topmost layer to remove the interactions due to periodic boundary conditions. The optimization was performed at a $3 \times 3 \times 1 \mathrm{k}$-point mesh for Brillouin zone integration, which was generated by the $\Gamma$-centered Monkhorst-Pack method. ${ }^{[55]}$ The gas-phase calculations of molecular structures were performed at wB97XD/6-311+G** [56] level of theory using the Gaussian 16 suite of programs. ${ }^{[57]}$ The QTAIM and NCI results were analyzed by the Multiwfn. ${ }^{[58]}$ The visualization of these analysis was using VMD. ${ }^{[59]}$ 

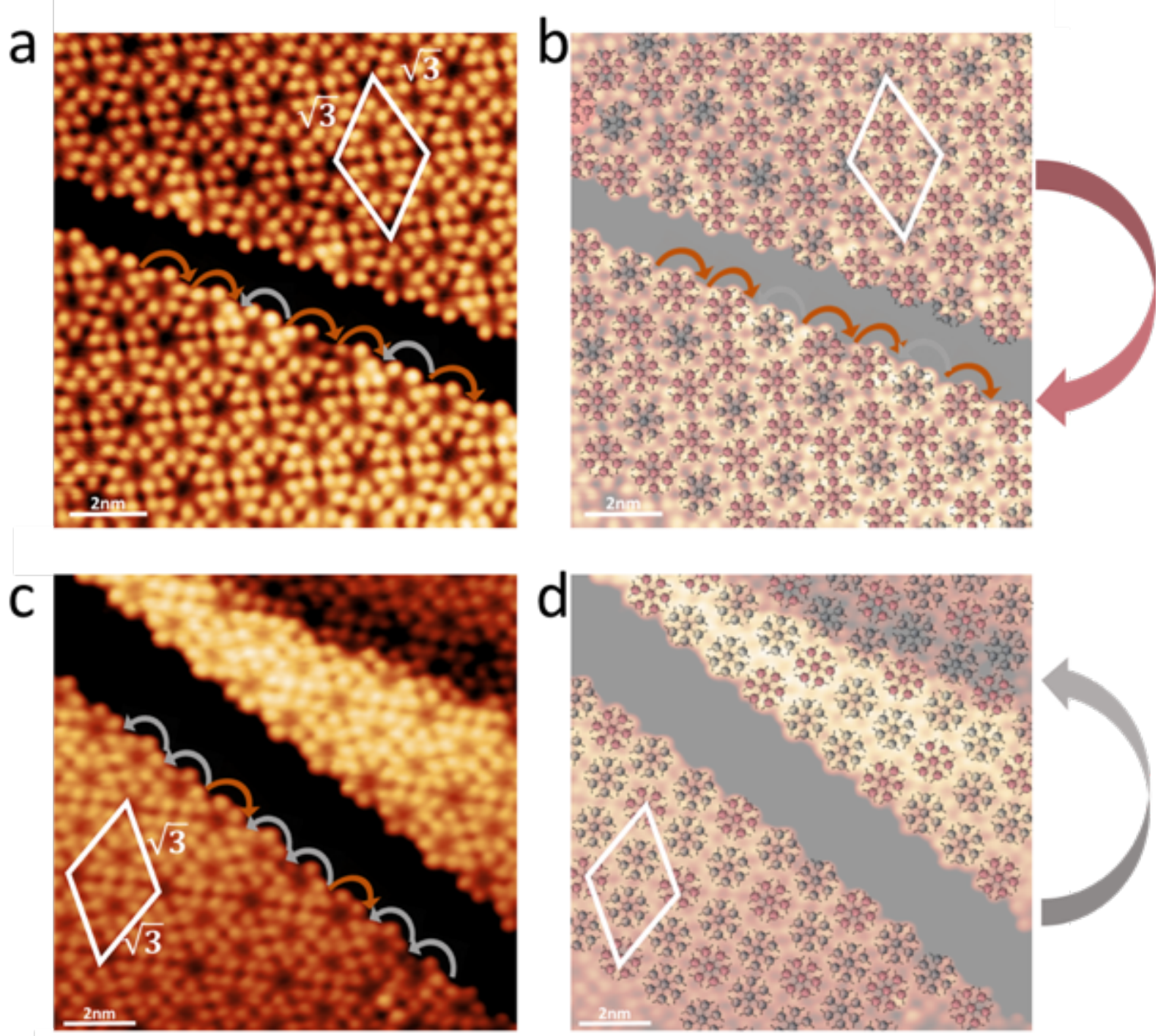

Fig. S1. Additional STM images of HPB self-assemblies. a, STM image of the HPB assembly with net L- long-range superlattice chirality. b, image in panel a superimposed with chemical structures of HPB molecule. c, STM image of the HPB assembly with net R- long-range superlattice chirality. d, image in panel c superimposed with chemical structures of HPB molecule. Grey and pink molecule structures denote L- handed and R- handed HPB enantiomers, respectively. 

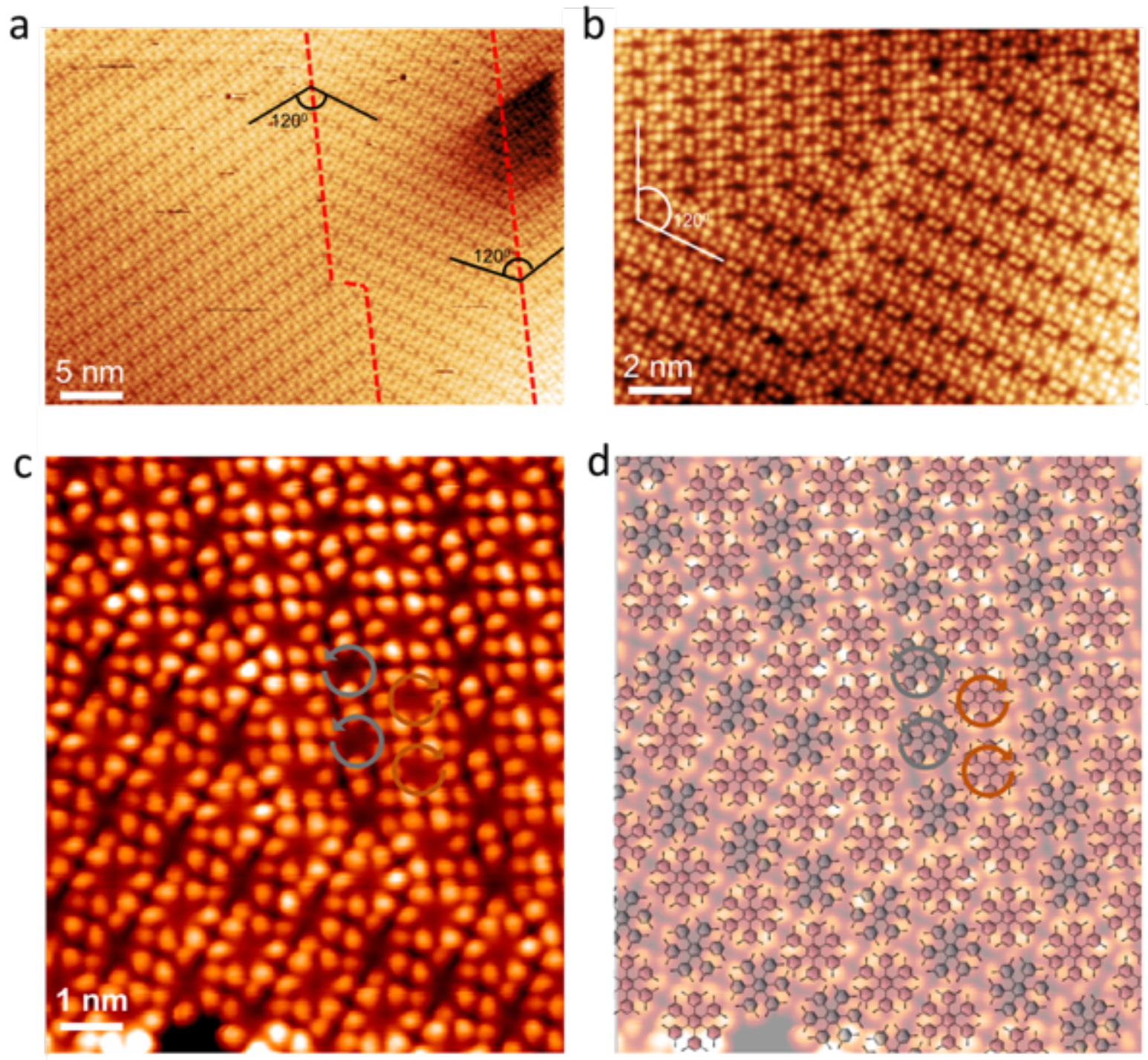

Fig. S2. Additional STM images of F-HPB self-assemblies. a, Large-scale image of F-HPB assembly shows a coexistence of multiple $2 \times 1$ domains and domain boundaries. $\mathbf{b}$, highresolution image of F-HPB assembly reveals the presence of multiple $2 \times 1$ domains co-existed with the $\sqrt{3} \times \sqrt{3}$ phase. c, high-resolution image highlights the L- and R-enantiomers, $\mathbf{d}$, image in panel c superimposed with chemical structures of F-HPB molecules. Grey and pink molecule structures denote L- handed and R- handed F-HPB enantiomers, respectively. 

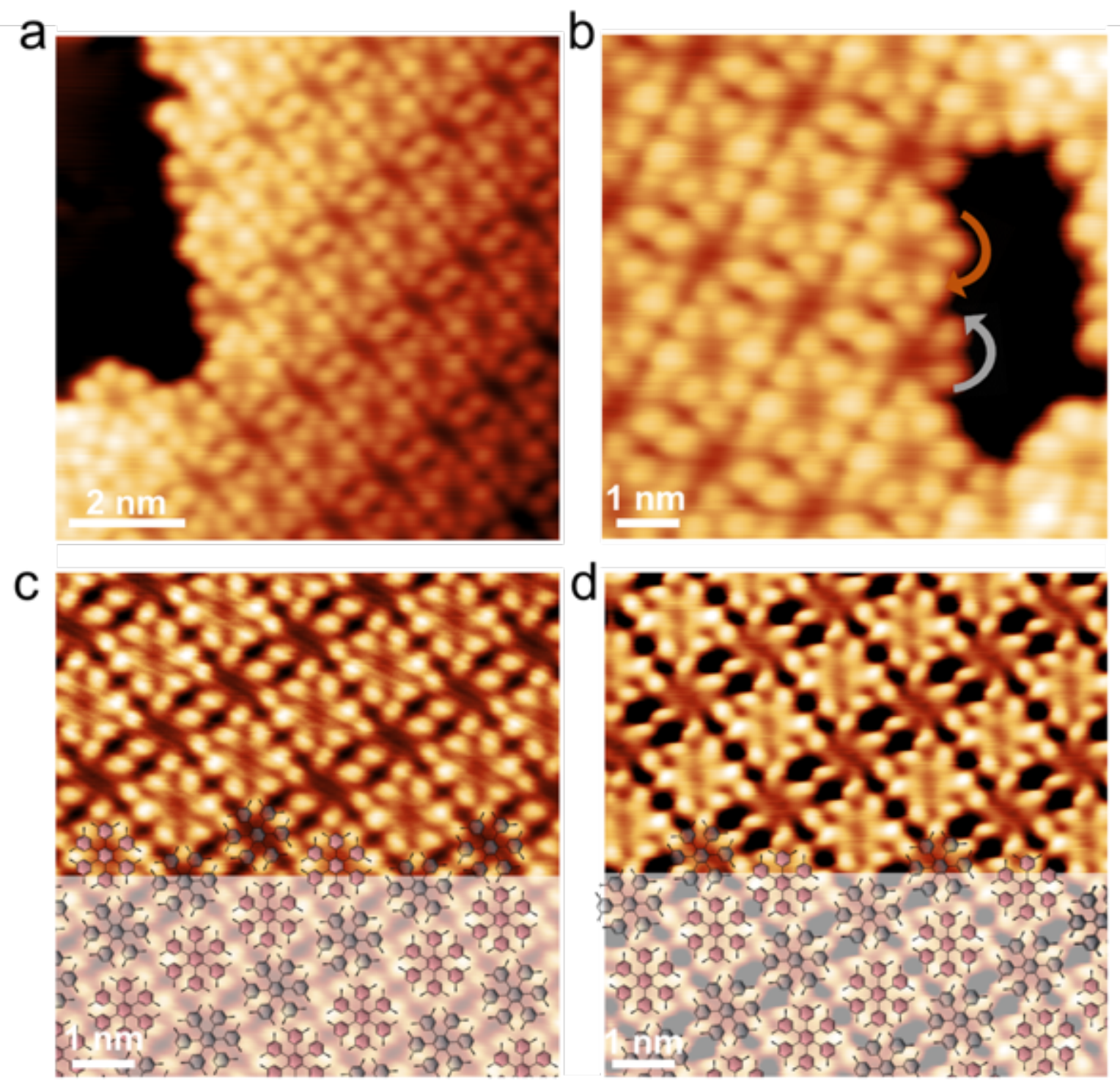

Fig. S3. Additional STM images of F-HPB self-assembly. a, b STM images of self-assembled monolayer F-HPB islands with edges. c, d, STM images acquired at different tunneling parameters: $V_{S}=1 \mathrm{~V}, I=0.5 \mathrm{nA}\left(\right.$ panel c) and $V_{S}=0.1 \mathrm{~V}, I=1 \mathrm{nA}($ panel d). Grey and pink molecule structures denote L- handed and R- handed F-HPB enantiomers, respectively. 
Table S1. The energy per molecule of HPB/FHPB $\sqrt{3} \times \sqrt{3}$ and $2 \times 1$ superstructures.

\begin{tabular}{|c|c|c|}
\hline Opt. single point energy (eV) & $\sqrt{3} \times \sqrt{3}$ & $2 \times 1$ \\
\hline HPB & -690.9654 & -690.8591 \\
\hline F-HPB & -692.8607 & -692.9254 \\
\hline
\end{tabular}

Table S2. Cohesive energies of the heterochiral and homochiral enantiomers is HPB $\sqrt{3} \times \sqrt{3}$ and F-HPB $2 \times 1$ structure

\begin{tabular}{|c|c|c|c|c|c|}
\hline \multirow{2}{*}{ Unit cell } & \multicolumn{2}{|c|}{ HPB } & \multicolumn{3}{c|}{ F-HPB } \\
\cline { 2 - 6 } & heterochiral & homochiral & homochiral-L & heterochiral & homochiral-R \\
\hline $\begin{array}{c}\text { Cohesive energy } \\
(\mathrm{kJ} / \mathrm{mol})\end{array}$ & -48.91 & -29.3 & -22.6 & -43.39 & -19.1 \\
\hline $\begin{array}{c}\mathrm{BSSE} \text { corrected } \\
(\mathrm{kJ} / \mathrm{mol})\end{array}$ & -45.77 & -26.4 & -19.8 & -38.45 & -16.8 \\
\hline
\end{tabular}



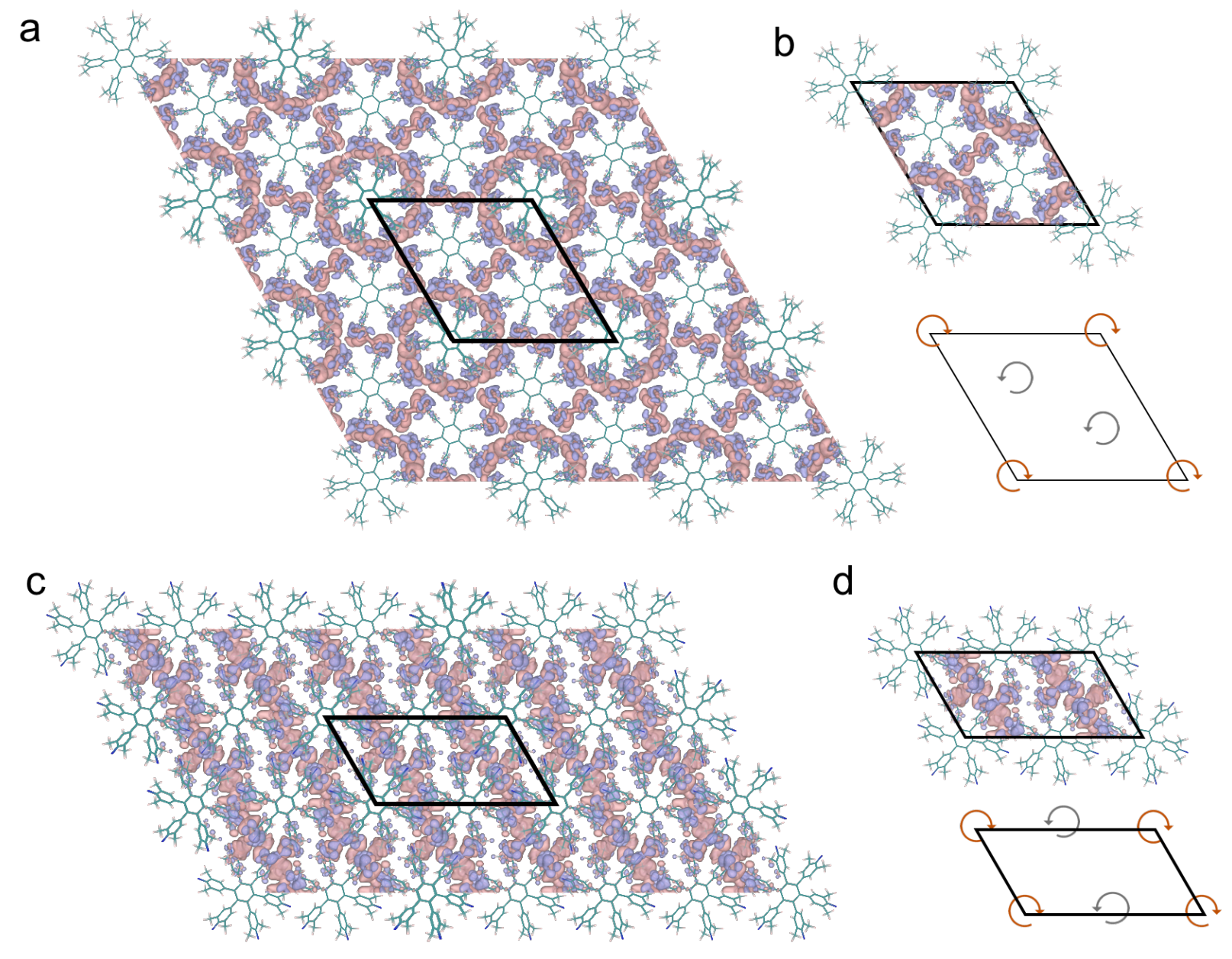

Fig. S4. Electrostatic potential in HPB and F-HPB assemblies. a, b Charge density difference (CDD) plots (panel a) and zoom-in image (panel b) of one unit cell the HPB assembly (c, d) Charge density difference (panel c) and zoom-in image of one unit cell (panel d) of the F-HPB assembly. The accumulation and depletion of negative charge is denoted by pink and purple color, respectively. Both CDD plots were generated at an isosurface of $0.0001 \mathrm{e} / \AA^{3}$ 


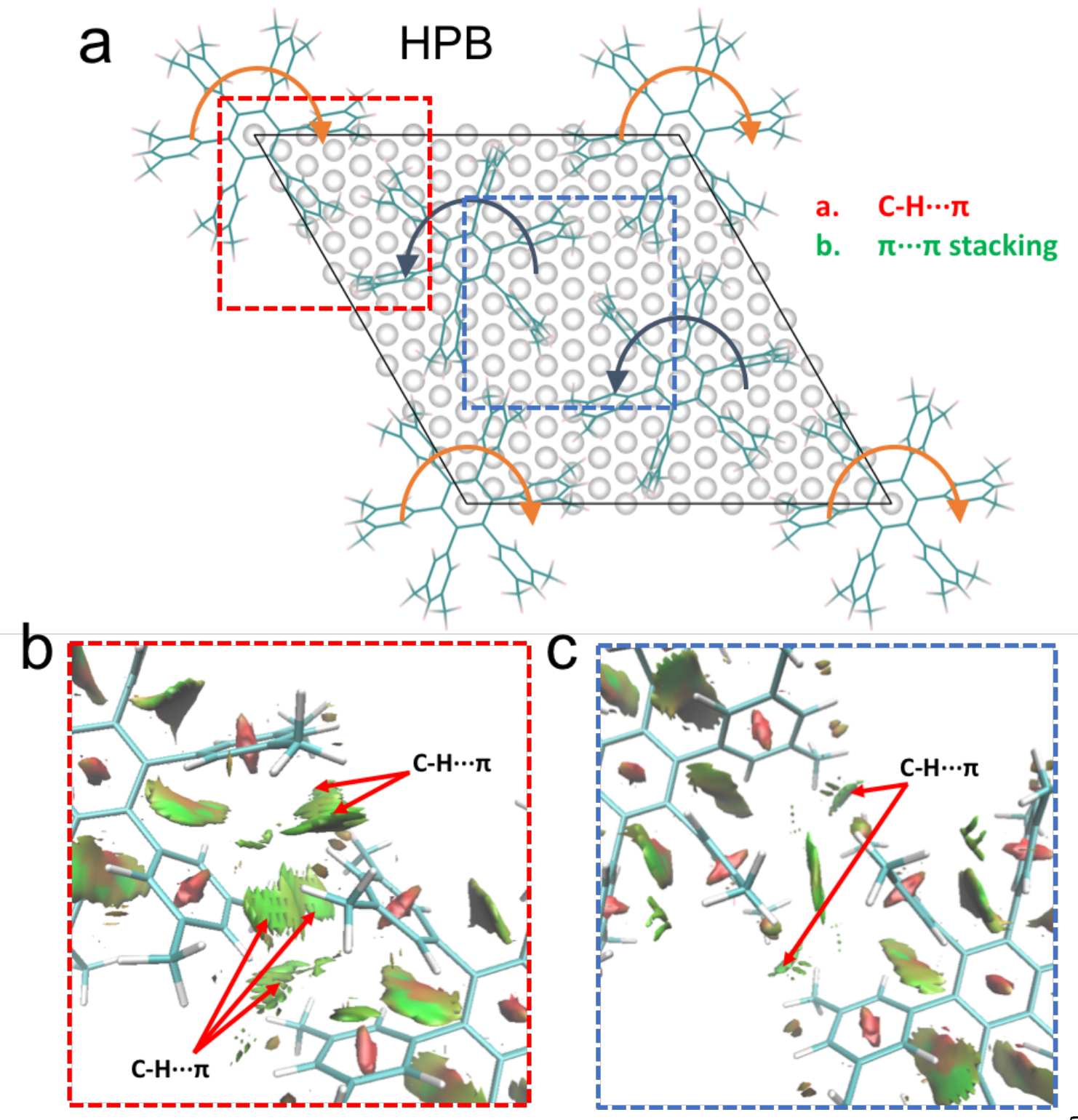

Fig. S5. Weak non-covalent interactions in HPB assembly. a, DFT-relaxed $\sqrt{3} \times \sqrt{3}$ elementary unit cell of the HPB assembly. $(\mathbf{b}, \mathbf{c})$ NCI plot of magnified view of the area marked by red (panel b) and red rectangle (panel c), revealing multiple weak $\mathrm{C}-\mathrm{H} \cdots \pi$ interactions between heterochiral HPB enantiomers (denoted as $\mathrm{a}_{1}-\mathrm{a}_{5}$ ). The weak NCI are represented by the discs. The color of the discs ranging from blue (strong attraction) to red (strong repulsion). The results are consistent with the AIM results. 


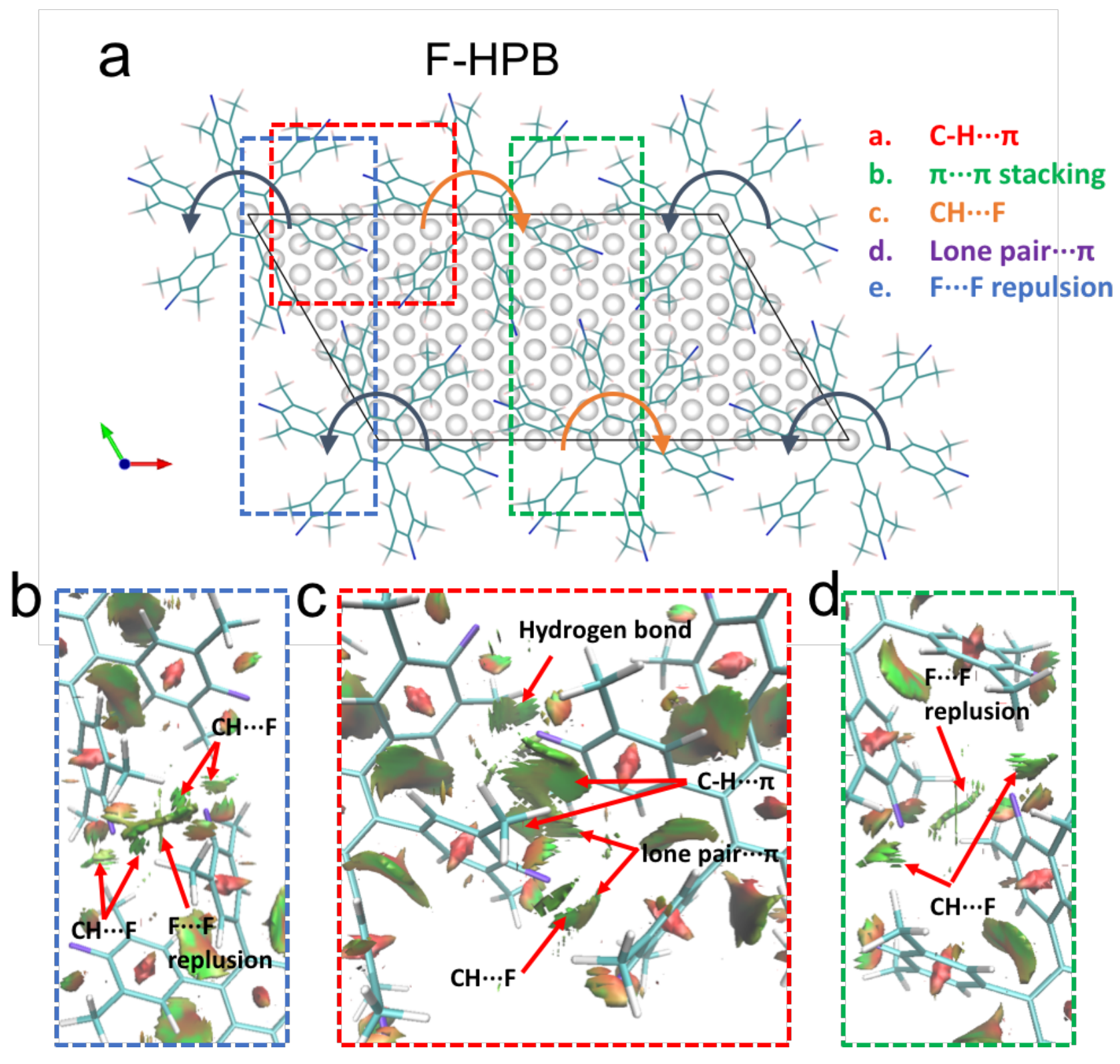

Fig. S6. Weak non-covalent interactions in F-HPB assembly. a, DFT-relaxed $2 \times 1$ elementary unit cell of the F-HPB assembly. (b, c, d) NCI plot of magnified view of the area marked by blue (panel b), red (panel c) and green (panel d) rectangle reveals a set of weak NCI between heterochiral F-HPB enantiomers including $\mathrm{C}-\mathrm{H} \cdots \pi$ (denoted as $\left.\mathrm{a}_{1}, \mathrm{a}_{2}\right), \mathrm{C}-\mathrm{H} \cdots \mathrm{F}\left(\mathrm{b}_{1}-\mathrm{b}_{3}\right)$ and $\mathrm{F}$ Lone pair $\cdots \pi\left(c_{1}, c_{2}\right)$ 
Table S3. Calculated topological properties of the electron density for the intermolecular interaction for HPB $\sqrt{3} \times \sqrt{3}$ structure

\begin{tabular}{|c|c|c|c|c|c|}
\hline \multicolumn{5}{|c|}{ heterochiral } & \multicolumn{3}{|c|}{ homochiral } \\
\hline BCP & $\rho(\mathrm{r})$ & $\begin{array}{c}\text { Laplacian } \\
\rho(\mathrm{r})\end{array}$ & $\mathrm{BCP}$ & $\rho(\mathrm{r})$ & $\begin{array}{c}\text { Laplacian } \\
\rho(\mathrm{r})\end{array}$ \\
\hline $\mathrm{a} 1$ & 0.00194 & 0.00571 & $\mathrm{a} 1$ & 0.00075 & 0.00239 \\
\hline $\mathrm{a} 2$ & 0.00297 & 0.00878 & $\mathrm{a} 2$ & 0.00075 & 0.00238 \\
\hline $\mathrm{a} 3$ & 0.00432 & 0.01278 & $\mathrm{~b} 1$ & 0.00206 & 0.00531 \\
\hline $\mathrm{a} 4$ & 0.00295 & 0.00868 & & & \\
\hline $\mathrm{a} 5$ & 0.00189 & 0.00556 & & & \\
\hline
\end{tabular}

Table S4. Calculated topological properties of the electron density for the intermolecular interaction for F-HPB $2 \times 1$ structure

\begin{tabular}{|c|c|c|c|c|c|c|c|c|}
\hline \multicolumn{9}{|c|}{ F-HPB } \\
\hline \multicolumn{3}{|c|}{ homochiral-L } & \multicolumn{3}{c|}{ heterochiral } & \multicolumn{3}{|c|}{ homochiral-R } \\
\hline BCP & $\rho(\mathrm{r})$ & $\begin{array}{c}\text { Laplacian } \rho \\
(\mathrm{r})\end{array}$ & $\mathrm{BCP}$ & $\rho(\mathrm{r})$ & Laplacian $\rho(\mathrm{r})$ & $\mathrm{BCP}$ & $\rho(\mathrm{r})$ & $\begin{array}{l}\text { Laplacian } \\
\rho(\mathrm{r})\end{array}$ \\
\hline $\mathrm{c} 1$ & 0.00372 & 0.01791 & $\mathrm{a} 1$ & 0.00673 & 0.0215 & $\mathrm{c} 1$ & 0.00531 & 0.0243 \\
\hline $\mathrm{c} 2$ & 0.00605 & 0.02721 & $\mathrm{a} 2$ & 0.00699 & 0.02188 & $\mathrm{c} 2$ & 0.00526 & 0.02413 \\
\hline $\mathrm{c} 3$ & 0.00611 & 0.02746 & $\mathrm{c} 1$ & 0.00748 & 0.03351 & $\mathrm{e} 1$ & 0.00104 & 0.00715 \\
\hline $\mathrm{c} 4$ & 0.00363 & 0.01748 & $\mathrm{c} 2$ & 0.00635 & 0.02924 & & & \\
\hline $\mathrm{e} 1$ & 0.00266 & 0.01678 & $\mathrm{~d} 1$ & 0.00278 & 0.01211 & & & \\
\hline & & & $\mathrm{d} 2$ & 0.0052 & 0.02297 & & & \\
\hline
\end{tabular}




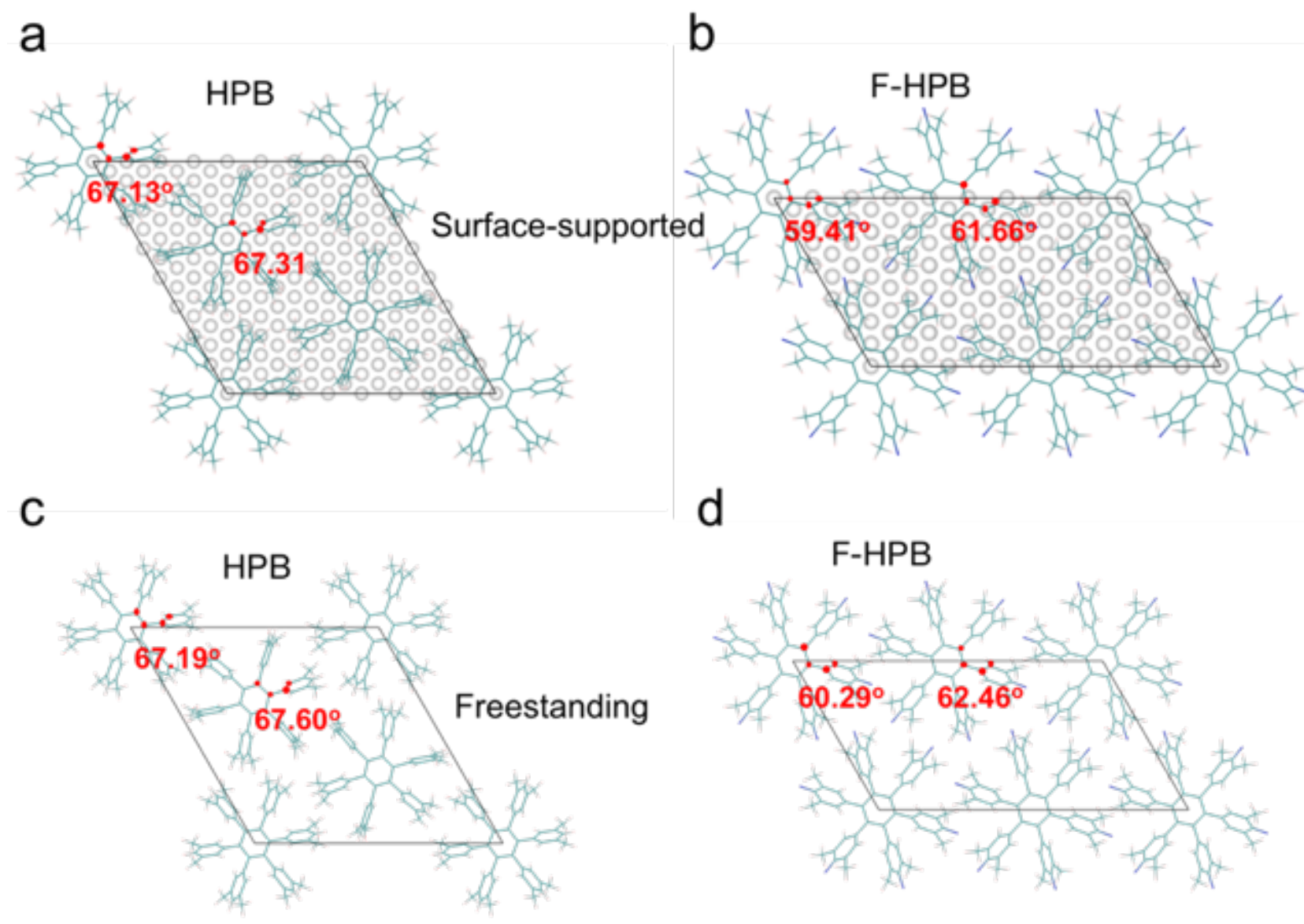

Fig. S7 (a, b) DFT-relaxed $\sqrt{3} \times \sqrt{3}$ elementary unit cell of the HPB assembly (panel a) and $2 \times 1$ unit cell of the F-HPB assembly (panel b) supported on four layers of $\operatorname{Ag}(111)$. (c, d) DFT-relaxed freestanding $\sqrt{3} \times \sqrt{3}$ elementary unit cell of the HPB assembly (panel c) and freestanding $2 \times 1$ unit cell of the F-HPB assembly (panel d). The values of dihedral angles are indicated on each panel.

\section{References}

[52] J. P. Perdew, K. Burke, M. Ernzerhof, Phys. Rev. Lett. 1996, 77, 3865-3868.

[53] P. E. Blöchl, Phys. Rev. B 1994 50, 17953-17979.

[54] S. Grimme, J. Antony, S. Ehrlich, and S. Krieg, J. Chem. Phys. 2010, 132, 154104.

[55] H. J. Monkhorst, J. D. Pack, Phys. Rev. B 1976, 13, 5188-5192.

[56] J.-D. Chai,M. Head-Gordon, Phys. Chem. Chem. Phys. 2008, 10 6615-20 (2008)

[57] M. J. Frisch, Gaussian 16, revision C.01 2016 Gaussian Inc., Wallingford, CT. 
[58] T. Lu, F. Chen, J. Comput. Chem. 2012, 33, 580-592.

[59] W. Humphrey, A. Dalke, K. Schulten, J. Mol. Graph. 1996, 14, 33-38 (1996). 